\title{
Understanding International GNC Hardware Trends
}

\author{
Adam Greenbaum \\ The Charles Stark Draper \\ Laboratory \\ 555 Technology Square \\ Cambridge, MA 02139 \\ 617-258-2736 \\ Agreenbaum@draper.com \\ Stephen P. Airey \\ ESA/ESTEC \\ Postbus 299 \\ 2200AG Noordwijk, The \\ Netherlands \\ +31 (0)71-565-5295 \\ Stephen.Airey@esa.int
}

\author{
Tye Brady \\ The Charles Stark Draper \\ Laboratory \\ 555 Technology Square \\ Cambridge, MA 02139 \\ 617-258-2366 \\ Tye@draper.com \\ Evan Roelke \\ Brown University \\ 69 Brown Street Box 4673 \\ Providence, RI 02912 \\ 908-698-9194 \\ Eval_Roelke@Brown.edu
}

\author{
Cornelius Dennehy \\ NASA Engineering \& Safety \\ Center \\ Goddard Space Flight Center \\ Greenbelt, MD 20771 \\ 301-286-5696 \\ Cornelius.j.dennehy@nasa.gov \\ Samuel Brady Judd \\ Massachusetts Institute of \\ Technology \\ 410 Memorial Drive \\ Cambridge, MA 02139 \\ Samjudd@mit.edu
}

\begin{abstract}
An industry-wide survey of guidance, navigation and control (GNC) sensors, namely star trackers, gyros, and sun sensors was undertaken in 2014, in which size, mass, power, and various performance metrics were recorded for each category. A multidimensional analysis was performed, looking at the spectrum of available sensors, with the intent of identifying gaps in the available capability range. Mission types that are not currently well served by the available components were discussed, as well as some missions that would be enabled by filling gaps in the component space. This paper continues that study, with a focus on reaction wheels and magnetometers, as well as with updates to the listings of star trackers, gyros, and sun sensors. Also discussed are a framework for making the database available to the community at large, and the continued maintenance of this database and the analysis of its contents.
\end{abstract}

\section{TABLe OF ConTents}

1. INTRODUCTION 1

2. THE DATABASE 1

3. RESULTS 2

4. DISCUSSION 7

REFERENCES ......................................................... 8

BIOGRAPHY. 9

\section{INTRODUCTION}

As part of the European Space Agency's (ESA) harmonization process, ESA is regularly tasked with obtaining a clear picture of the available and state of the art attitude determination and control subsystem (ADCS) hardware worldwide. This is then used, along with future mission needs, to put ESA's development roadmap into context. Databases of available ADCS equipment are also regularly called for during mission feasibility, pre-phase A and phase A studies. NASA has similar needs for a clear picture of the current status of equipment supply within the
United States (US) and the availability of non-US products for their own missions. In addition to the needs of the Agencies, databases on available hardware are used by industry for their own tradeoffs for both commercial and institutional missions.

While such databases are in principle rather simple and far from an advancement in high technology, they require a lot of work to set up and maintain, become rapidly out of date, and frequently suffer from missing or conflicting data. Further, each individual entity (NASA, ESA, industry) has its own limitations (be it insight, manpower, or both) in data collection for the population of such databases. With both NASA and ESA needing to update their databases at the same time, it was decided to combine the efforts and work together to assemble a common database that would be more complete than available time and effort would allow either to produce on their own.

This collaborative database population work follows from preliminary work performed in 2014 by the NASA Engineering Safety Center in collaboration with Draper laboratory and a previous version of the ESA database. This work has been extended in scope both by of the number of units included and in efforts to include historical data and products from outside of the European Union (EU) and the US.

\section{The DATABASE}

Due to the international makeup of the group performing this work (and similarly, the likely user base), some thought must be devoted not only to US export laws, but also EU laws and company privacy/security issues. In order to be publishable without restriction (ITAR, EAR, or proprietary data), all data collection was from publically available sources only. In other words, if it could not be found on the internet, it was not included in the database. Further, strong preference was given to company-published datasheets whenever these were available. Occasionally, it was required to make use of other sources such as journal articles or 
company websites. In all cases, a copy of the original source information was taken and is linked to the database.

The database is currently populated with star trackers, sun sensors, gyros, magnetometers, and reaction wheels; although both NASA and ESA have interest in expanding this further in future work to include magnetic torquers, earth sensors, control moment gyros, and global navigation satellite system receivers.

Various metrics were recorded for each class of component. These metrics were selected not only to aid in identifying technology trends and technology planning, but also to be useful to spacecraft engineers performing trade studies and selecting hardware for missions. Key performance metrics (dependent on type of hardware), mass, power consumption, and interface information were included. Other metrics were added to aid sorting and searching such as the maturity/flight heritage as well as links to the information sources.

The information was recorded in spreadsheet format as a Google Spreadsheet. This was done to allow multiple contributors to enter and edit information simultaneously, an essential capability given multiple parties on opposite sides of the Atlantic were contributing.

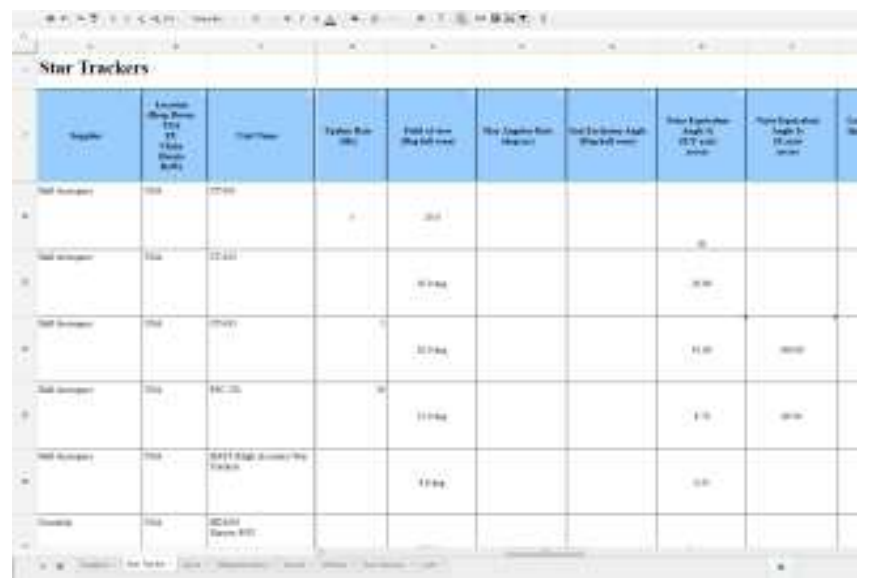

Figure 1 - A Screenshot of the database. In many cases, not all metrics were published or available to be incorporated.

As can be seen in Figure 1, not all fields are populated. There is great variability in the amount and type of information published in specification sheets across various suppliers, and sometimes even within a single supplier, with many missing key information on their product. As a result, we have focused our analysis on parameters for which we have better coverage.

Additional difficulties arise due to acquisitions within the aerospace industry. In some cases, the same physical sensor or actuator was entered into the database twice, under both the original manufacturers name and under the company that acquired them. These duplicate entries were removed from the database as they were discovered.
Analysis and the generation of plots were typically performed with a copy of the database exported to Microsoft $^{\circledR}$ Excel $^{\circledR}$.

\section{RESULTS}

\section{Previous Findings}

In the previous study performed by NASA and Draper (2013-2014), which served as a starting point for the current work, several regional trends were observed and the additional data collected agree with these previous conclusions. The initial findings are summarized in the next few paragraphs. For a more detailed discussion of these findings, see reference [1].

Within the gyroscope market, the full spectrum of performance is well represented with clear dominance in both performance and availability of sensors produced in the US. Micro electro-mechanical sensor and fiber-optic gyro sensors continue to improve and enter market segments previously dominated by heavier mechanical systems.

Within the star tracker market, the EU showed a clear dominance in performance and availability of sensors. It is the opinion of the authors that this is largely due to the effectiveness of ESA's road mapping and technology planning, along with reliable long-term funding for such programs. In terms of technology trends, a new generation of lightweight star trackers began to appear on the market, prioritizing low mass and power over accuracy.

Additionally, several gaps in the star tracker market place were identified and some thoughts were expressed regarding missions that could be enabled by filling those gaps.

\section{Trends in Interfaces}

The database can also be used to infer trends on Avionics and to assist in data handling architecture trade offs. In 2009, there was a paper presented to the ESA Avionics, Data, Control and Software systems (ADCSS) Workshop entitled 'AOCS Interfaces Working Group - Purpose and Progress'. This reported on work performed within the Savoir Advisory Group (a collection of industry and ESA that provide advice on Avionics issues) to investigate data and power interface trends with the goal of setting the direction for the rationalization of power and data interface requirements for future developments as the proliferation of different interfaces was seen as a key cost driver for both supplier and system prime and as a barrier to competition between unit suppliers.[2]

The approach followed for that investigation was primarily a survey-based approach of a representative cross section of suppliers, buyers, and on-board computer manufacturers (on whom there is a large impact); and as such, relied both on some hard knowledge and some element of market 
perception. This work can now be augmented by extracting interface information from the database.

Data Interfaces-The 2009 work indicated that the interfaces used amongst supplied ADCS hardware was dominated by MIL-1553 and RS-422/485 in rather equal measure (see Figure 2). Spacewire also had a noticeable market volume. This result was of course heavily influenced by European suppliers and their perception of the supply situation in the rest of the world.

Looking to the ADCS equipment database as it now stands and acknowledging that this is still missing information in some areas - one can see the actual supply situation (or at least 'availability' situation) for data interfaces (Figure 3). There are some notable differences.

\section{ADCS Interfaces (w/o Sun Sensors)}
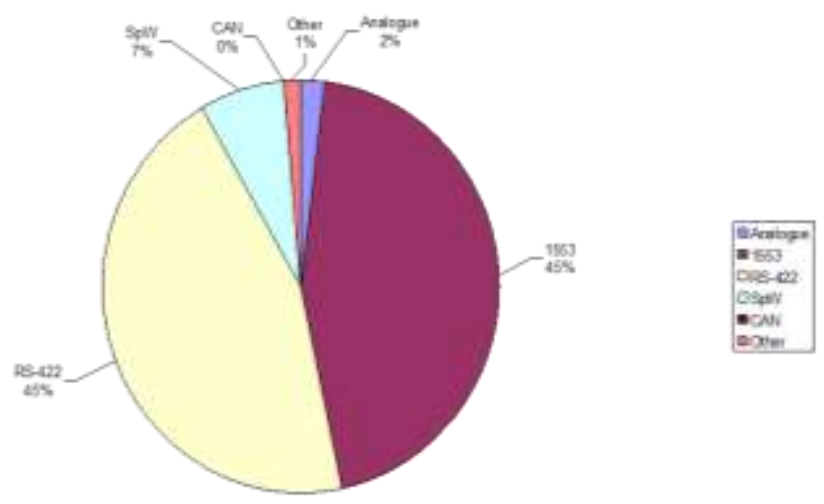

Figure 2 - Original assessment of perceived data interface supply based largely on European markets. From [2].

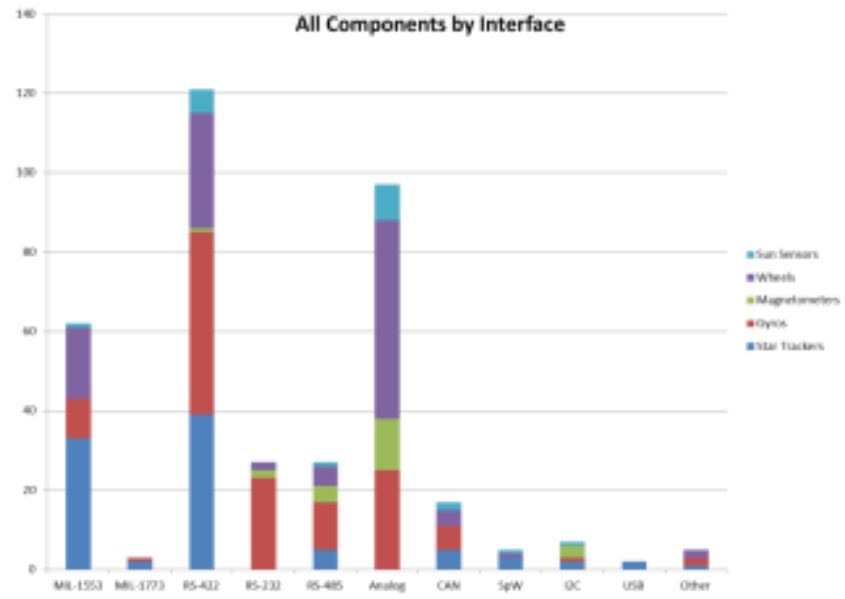

Figure 3 - Availability of different data interface support within the database which covers worldwide supply (units supporting multiple interfaces counted for each).

When combining RS-422 with the closely related RS-485 it is clear that this largely dominates the available digital interfaces in the world market and is significantly more popular than MIL-1553 (see Figure 4). It is also clear that there are far more analogue interfaces still being supported than would be obvious from just looking at a European market, although a very large proportion of this is coming from reaction wheels where there are a lot of different size/configuration variants available. The sun sensor contribution is also likely to be underestimated in this plot as many suppliers seem not to explicitly include their supported interfaces on their datasheets.

Also noticeable is the relatively large number of CAN, I2C and 'Other' interfaces available although it does appear as though a large percentage of these are coming from units aimed clearly at cubesats and hence their relevance to the wider market is questionable.

Otherwise, in broad terms, the study presented in 2009 and the examination of the data gathered in this database lead to similar conclusions. Considering the widespread support of RS-4XX interfaces, a protocol standard for space seems clearly advantageous (see Figure 5). ESA has been working on a draft of such a protocol standard, at least for European missions and suppliers, since the 2009 study.

\section{Perceived Main Interface Choice by Market}

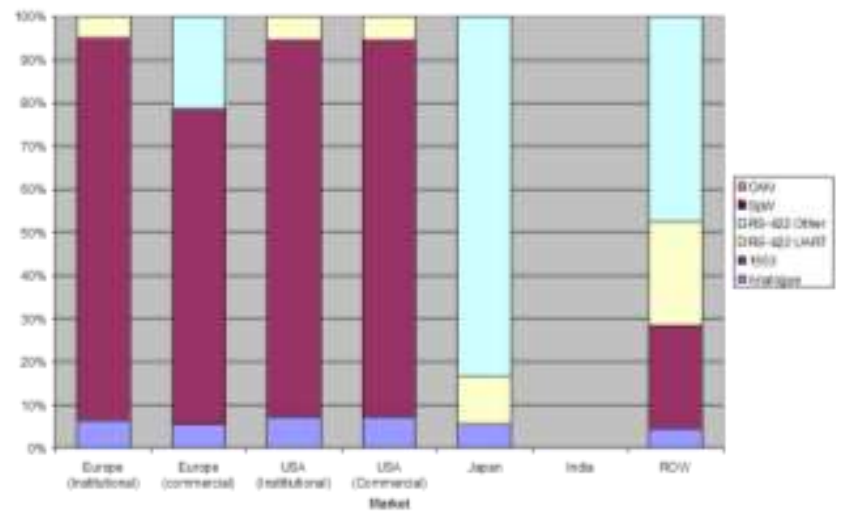

Figure 4 - Perceived main interface choice by market in 2009. MIL-1553 showed clear dominance. Reproduced from [2].

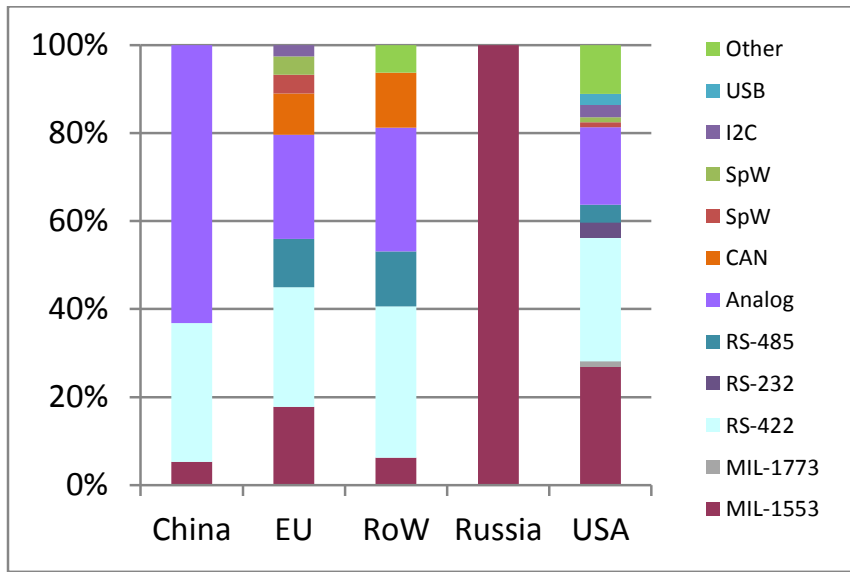

Figure 5 - Interface preference by region. Serial interfaces (RS-4XX) have become substantially more popular.

A further interesting point to note is that the US supports, by far, the most diverse range of data interface standards on ADCS equipment. While a significant contribution to this 
comes from equipment designed for cubesats, there is still a large spread and lack of standardization.

Power Interfaces-Comparing the 2009 study to the current data, there are some clear similarities. $28 \mathrm{~V}$ primary power was the dominant choice then, and has become more popular. The $50 \mathrm{~V}$ bus seems to have declined in popularity this may reflect the higher proportion of smaller satellites operating at lower voltages being launched today. Likewise, the 5 and $15 \mathrm{~V}$ voltages have increased in popularity (see Figure 6 and Figure 7).

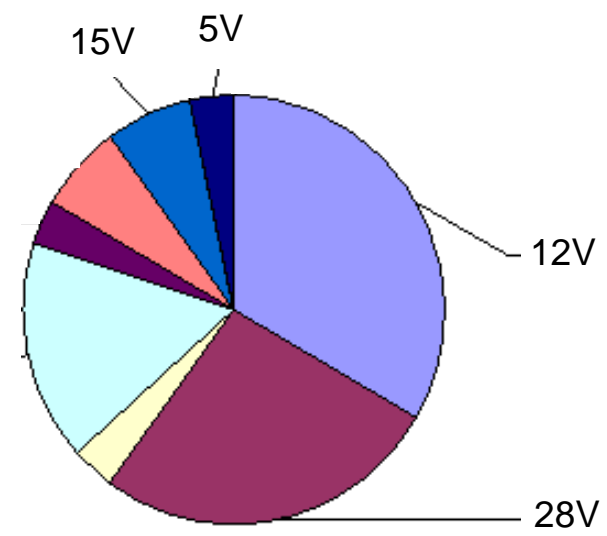

Figure 6 - Power interface preferences, reproduced from [2].

\section{Power interfaces}

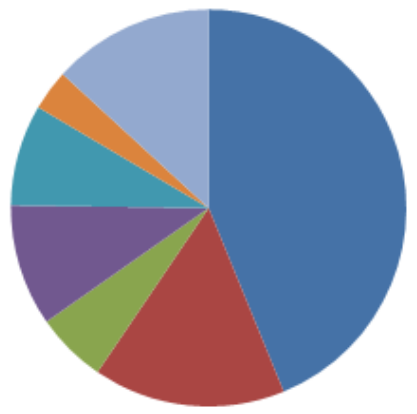

28V Prim
- 50V Prim
100V Prim
- 5V sec
15V sec
Negative Voltages

Figure 7 - Power interfaces as represented in the current database. Higher voltages have declined somewhat in popularity.

\section{Regional Trends}

As can be seen in Figure 8, The US produces the greatest number of products as captured by this survey, with the EU following closely. However, the EU has a larger number of companies providing spacecraft hardware, as shown in Figure 9.

\section{Different products per region (In production)}

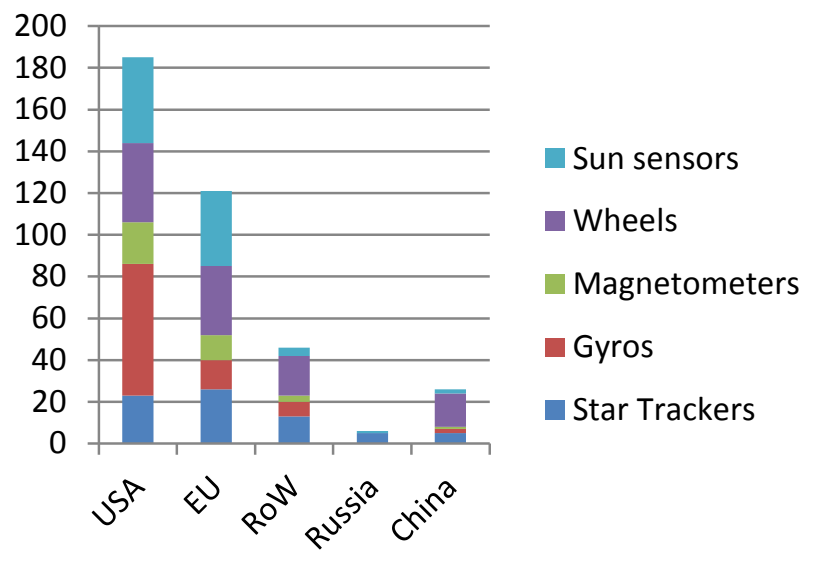

Figure 8 - Hardware produced by region, as represented in the database.

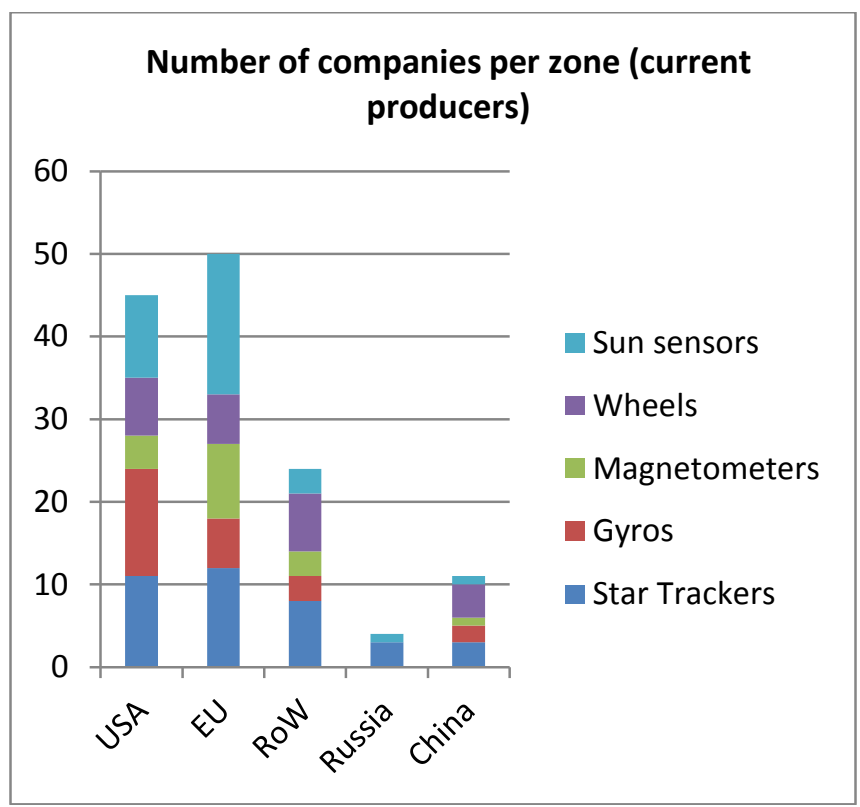

Figure 9 - Distinct companies in each region of interest. The EU has the greatest number of companies producing hardware.

\section{Reaction Wheels}

The state of the Reaction Wheel market, shown by the plots in Figure 10, is not encouraging, as the data clearly demonstrate that there are gaps in availability. The demise of Ithaco/Goodrich wheels has led to a lack of available wheels, US produced or otherwise, in the 4-8 N-m-s range. Additionally, in most size ranges (particularly on the smaller end), only a single US supplier exists, which is worrying. In the EU, this situation is slightly better, with models from two suppliers available across a variety of sizes. 


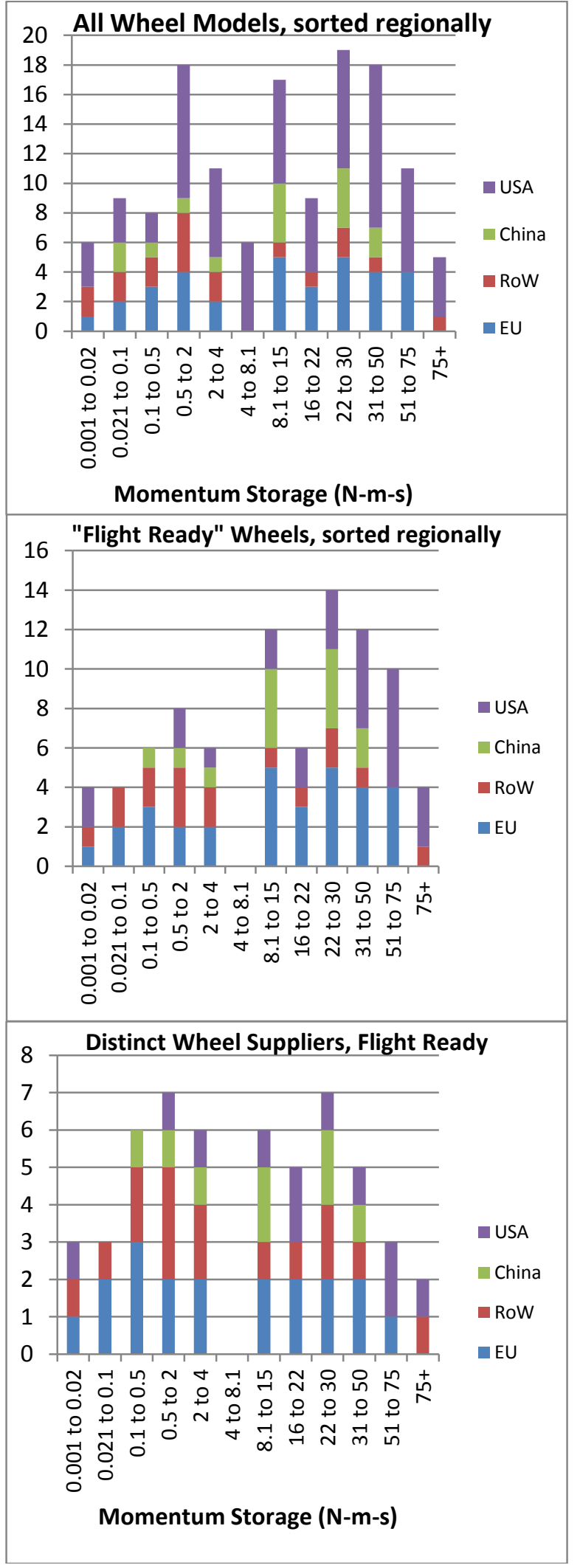

Figure 10 - (a) Number of wheel models available by size range. (b) Subset of these wheels that are deemed "flight ready". (c) Distinct manufacturers of "flight" ready wheels. In many cases, only a single manufacturer is producing reaction wheels in a given size range.
As shown in Figure 11, plotting momentum storage versus mass appears to indicate a clear threshold of performance. There are many wheels that ride along this threshold - those that do not are either old models out of production or are high-torque designs. There are 4 wheels that surpass this threshold - one of which clearly has an error on the spec sheet. The others were advertised by a company that no longer exists and likely never produced flight hardware. These wheels utilized carbon-composite rotors, indicating that it may be worth investigating the source of the current performance threshold, and whether lighter and stronger rotors may be of benefit, or if this is a red herring. This plot shows that momentum storage as a function of unit mass is unlikely to become a key selling point, as it is highly physics limited, and that to out-sell other products, reliability, cost, and other performance characteristics will be the likely drivers.

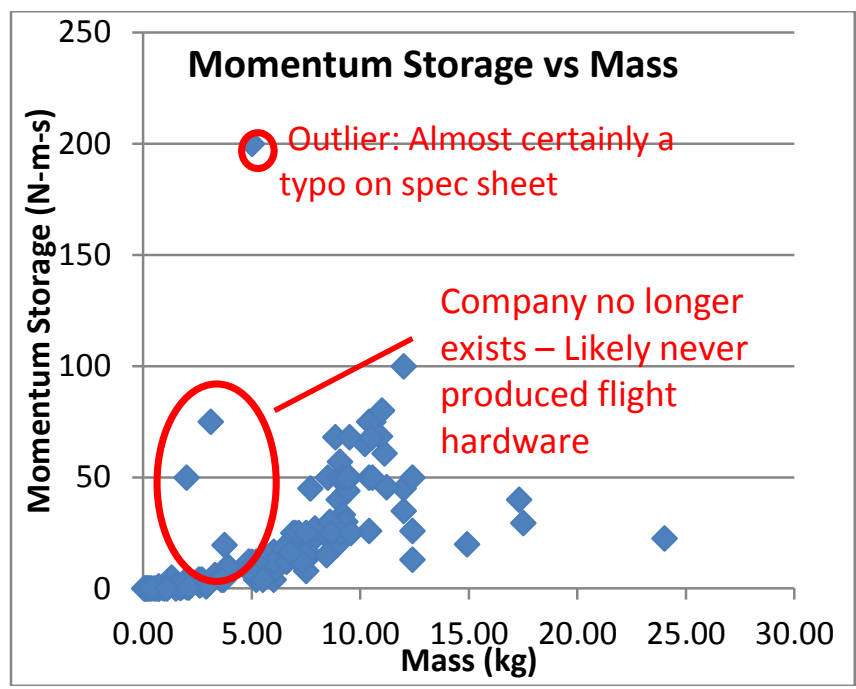

Figure 11 - Momentum storage capacity vs unit mass for reaction wheels. There appears to be a clear frontier of performance. Published specifications for products above the frontier line are suspect.

\section{Star Trackers}

The EU appears to continue to dominate the star tracker market, with multiple offerings across all ranges. There is some progress among US manufacturers in catching up, particularly on the lightweight end, but this has not resulted in any flight grade units as of yet. There is also a distinct and clear drive by Russia, China, and the rest of the world to chase down this European dominance, with efforts clearly being spent in developing star trackers to compete in the medium performance class (high volume) market sector (see Figure 12). 


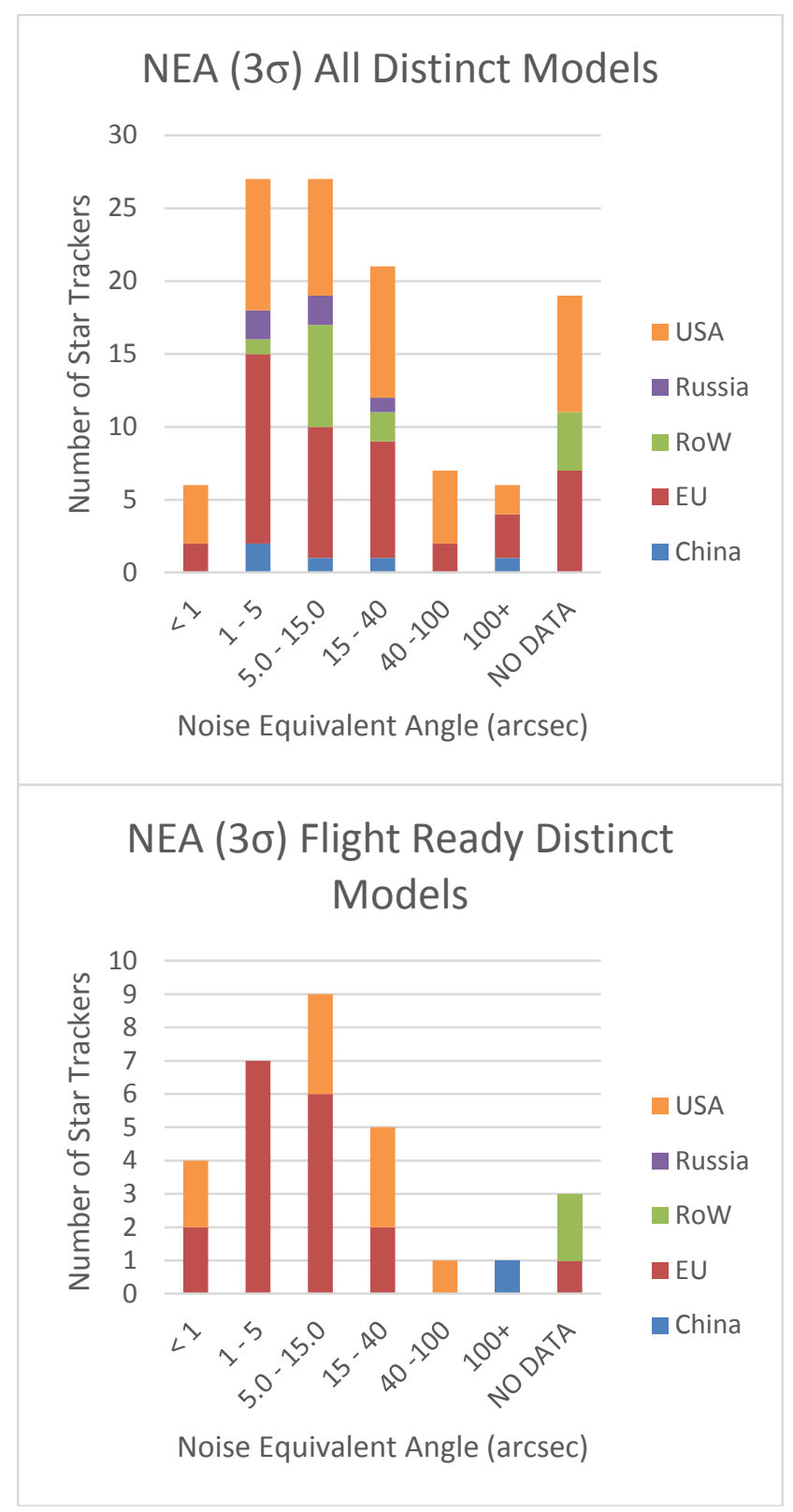

Figure 12 - The number of star trackers available in each performance category (top), and the subset of those that are flight ready (bottom). Noise Equivalent Angle (NEA) defines the performance categories

\section{Gyros}

There is continued dominance by US manufacturers in the Gyro market, but it is clear that the EU is under-represented in this survey. While the database contains several flightgrade gyros from the EU, many had insufficient information to be included in Figure 13, or did not have datasheets publicly available.

\section{ARW by Unique Flight Ready Device}

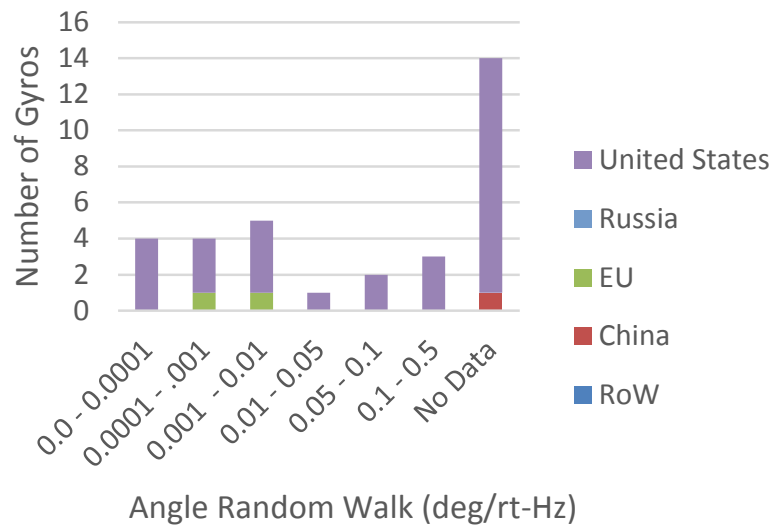

Figure 13 - Availability of gyros by performance category (Angle Random Walk, ARW).

\section{Magnetometers}

Perhaps the most striking result from the magnetometer survey is the large quantity of analog magnetometers (see Figure 14). Whereas most units seem to be transitioning to digital interfaces (or at least have significant digital interface options supported), this is not the case for magnetometers. The reason for this is not understood and this may therefore represent a market opportunity. Nearly as striking as the number of analog units on the market is the number of units that do not specify their interface on the datasheet.

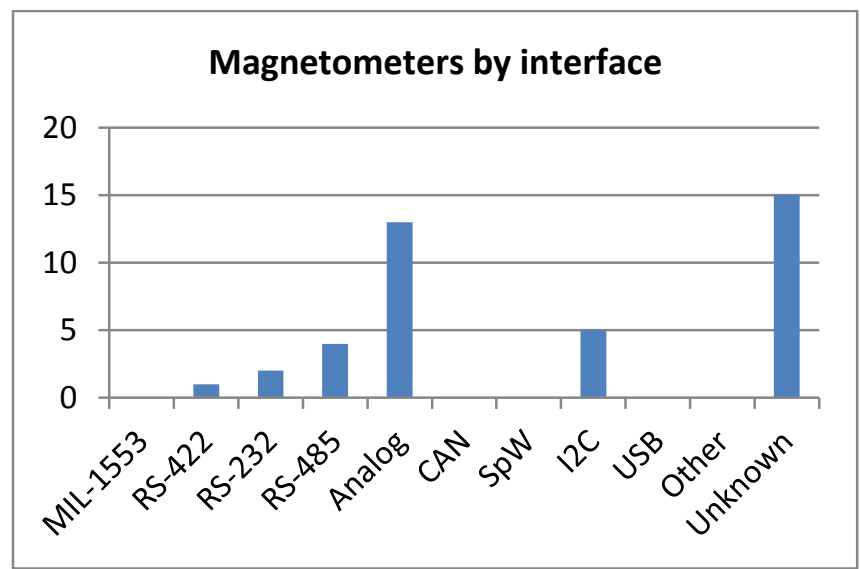

Figure 14 - Magnetometers by interface type

A comparison of magnetometer performance is not terribly instructive, as most magnetometers have noise levels orders of magnitude lower than the Earth magnetic field in low Earth orbit. As a result, almost any magnetometer can meet the requirements imposed by basic ADCS needs, and other factors (such as price and interface) will likely guide selection. 


\section{Sun Sensors}

In the sun sensor market, there is a large supply of analog units, as is to be expected. Among digital units, serial interfaces dominate. The most striking feature with regards to the information in the database is the sparseness of interface information, as can be seen in Figure 15. This is an artifact of the information coming from sources other than spec sheets, such as conference and journal papers, which have largely incomplete information.

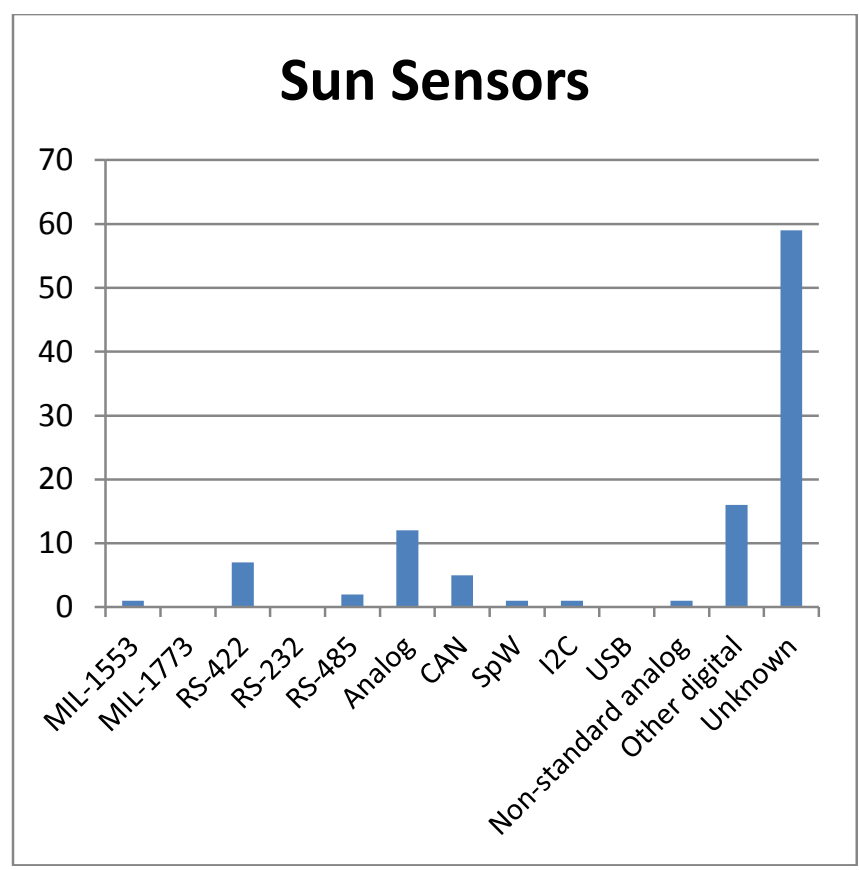

Figure 15 - Sun sensors by interface.

\section{Discussion}

\section{Known Shortcomings}

As previously mentioned, there were some difficulties encountered in the creation of the database and in the analysis of its contents. Not all datasheets contained all of the information required, and in some cases (particularly with Gyros) the information, if provided, was in nonstandard, or inconvenient units. Furthermore, some of the values listed were viewed as questionable - for example, on many gyro spec sheets, especially on the commercial/lower performance end, the bias stability values listed seemed very optimistic. In most cases no correlation time, or other factors pertinent to the measure of bias stability (i.e., environmental conditions) were listed, which significantly reduces the value of bias stability as a standard performance metric. For this reason, Angle-of Random-Walk (ARW) was the preferred metric when evaluating the performance of Gyros.

One noticeable shortcoming of the database is the underrepresentation of the EU in flight-grade gyros. The existence of these missing gyros is known to the authors from previous experience, but for a variety of reasons (proprietary information, missing flight heritage information), these sensors could not be included in any of the analysis presented earlier.

Another difficulty encountered concerned the collection of historical sensor data, which are useful in identifying trends. Many of these sensors are of pre-internet vintage. Information regarding these sensors in some cases came from paper copies of spec sheets in the possession of the authors, or journal papers and/or conference proceedings discussing their performance. In many cases, the information regarding these older components is incomplete.

Due to the expanded geographic coverage in this iteration of the database, language became an issue. Certain regions (mainly China and Russia) have far fewer components in the database than would be expected. We attribute this underrepresentation, at least partially, to the lack of publicly available information in English.

\section{Next Steps}

The immediate next step for this database is to host it on the NASA Engineering Network for NASA-internal use by GNC designers and spacecraft engineers. Publishing the database to a larger audience is planned, but there are some issues to be worked out. The largest obstacle for public release by NASA is concern over ITAR and proprietary data issues. While the data collection effort was specifically structured to avoid these issues, ITAR remains a NASA concern.

Ultimately, the vision for this database is that of a curated, open-source database that is a resource for the community. Participation by hardware vendors as well as users will directly address many of the shortcomings identified in the database as it currently exists. Hardware-specific templates (i.e., separate templates for star trackers, reaction wheels, etc.) submitted by vendors would go a long way towards addressing the issues of data completeness and homogeneity. Allowing the community at large to submit information (curated by database administrators) could provide missing information on hardware that is out of production, giving a complete chronological picture of the GNC hardware market, which would be useful in identifying trends.

Community involvement is also desired to aid in analyzing and understanding the database as it continues to grow. The analysis presented in this paper represents only a fraction of the total analysis completed, which was motivated by very specific goals. It is expected that we have only scratched the surface, and that the community will make use of this data using a variety of tools and methodologies for GNC technology planning, benchmarking, and trend identification.

\section{Conclusion}

In the year since the initial database was created, we have expanded both the categories of sensors catalogued and the 
number of sensors within those categories. With this data in hand, we have focused on finding gaps and identifying GNC hardware trends both by category and in the industry as a whole. Some of the gaps identified, such as those in the availability of reaction wheels, are worrying; particularly for the US space industry.

\section{REFERENCES}

[1] A. Greenbaum et al, "Finding the Gaps in Space GNC Hardware", 2014 IEEE Aerospace Conference, Big Sky, MT, 1-8 March 2014

[2] S.P. Airey et al, "AOCS Interfaces Working Group Purpose and Progress", 2009 ESA ADCSS Conference, Noordwijk, Netherlands 3 November 2009. 


\section{BIOGRAPHY}

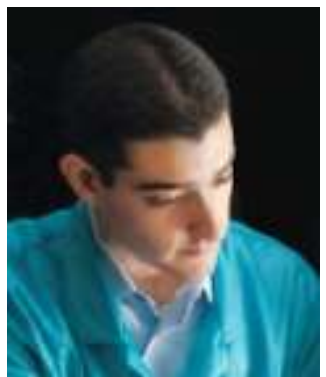

Adam Greenbaum is a Senior Member of the Technical Staff in the Space Systems Engineering Group at Draper Laboratory. His research interests include fault tolerant systems, mission design, propulsion, small spacecraft, remote sensing, and systems engineering. Since joining Draper, his projects include fault management modeling for the Orion Crew Vehicle, ExoplanetSat, and ADCS design for CYGNSS. $M r$. Greenbaum earned his $S c B$ in Mechanical Engineering, and his ScM in Solid Mechanics, both from Brown University. His masters thesis concerned the design of a small, responsive launch vehicle for nanosatellites.

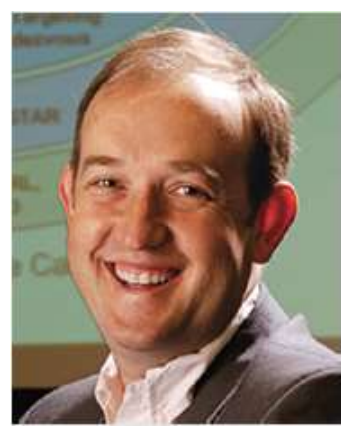

Tye Brady is a Distinguished Member of the Technical Staff and a Group Leader in the Space Systems Engineering Group at Draper Laboratory. He has worked over the past 26 years on spacecraft instrumentation, design, and integration on a wide variety of programs. He holds a BS in Aerospace Engineering from Boston University and a SM in Aeronautics and Astronautics from MIT.

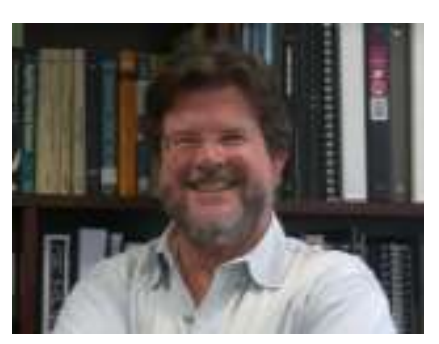

Neil Dennehy is the NASA Technical Fellow for Guidance, Navigation and Control. As a member of the NASA Engineering and Safety Center (NESC) he provides technical leadership for independent test and analysis, risk assessment and problem resolution for some of NASA's most complex $G N \& C$ problems. He has over 35 years' experience in the design, development, integration and operation of $G N \& C$ systems for communications, national security, Earth remote sensing and other scientific platform applications. His technical emphasis has been in the areas of spacecraft attitude determination and control system design, autonomous rendezvous and proximity operations, space system engineering, and $G N \& C$ technology development. Before joining the NESC organization he was the GN\&C Technologist at NASA's Goddard Space Flight Center. He holds a BS in Mechanical Engineering from the University of Massachusetts/Amherst and a SM in Aeronautics and Astronautics from MIT.
Stephen (Phil) Airey has been at ESA for the last 15 years where he has spent his time mainly in developing and improving GNC hardware, with emphasis on Star Trackers, Active Pixel Sensors and Reaction Wheels but with also supporting other equipment such as Sun Sensors, Gyros, Accelerometers and Magnetometers. He has provided hardware support to many ESA missions starting with work on XMM and INTEGRAL and is currently supporting the Fine Guidance Sensor development for the up coming Euclid mission.

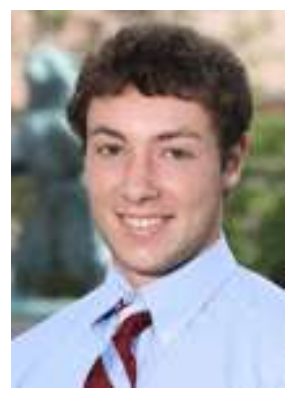

Evan Roelke is currently working towards a Bachelor's of Science in Aerospace Engineering at Brown University and is on track to graduate with Honors. After graduating in May 2015, Evan is interested in pursuing a PhD in Astrodynamics or Space Systems/Exploration. In addition to his studies, Evan is a member of Brown's Varsity Cross Country and Track\&Field programs. During track season Evan trains for the 3000 meter Steeplechase and the 5000 meter run. In his free time, Evan enjoys reading, listening to music, and outdoor activities.

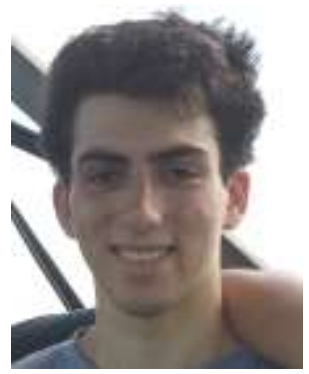

Sam Judd is a sophomore at MIT studying aerospace engineering. His research interests include propulsion, supersonic flight, GN\&C algorithms, and human spaceflight. 